\title{
Improving 1D Stellar Models with 3D Atmospheres
}

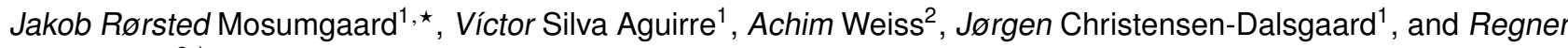 \\ Trampedach ${ }^{3,1}$ \\ ${ }^{1}$ Stellar Astrophysics Centre (SAC), Department of Physics and Astronomy, Aarhus University, Ny Munkegade 120, DK-8000 \\ Aarhus C, Denmark \\ ${ }^{2}$ Max-Planck-Institut für Astrophysik, Karl-Schwarzschild-Str. 1, D-85748 Garching, Germany \\ ${ }^{3}$ Space Science Institute, 4750 Walnut Street, Suite 205, Boulder, CO 80301 USA
}

\begin{abstract}
Stellar evolution codes play a major role in present-day astrophysics, yet they share common issues. In this work we seek to remedy some of those by the use of results from realistic and highly detailed 3D hydrodynamical simulations of stellar atmospheres. We have implemented a new temperature stratification extracted directly from the 3D simulations into the Garching Stellar Evolution Code to replace the simplified atmosphere normally used. Secondly, we have implemented the use of a variable mixing-length parameter, which changes as a function of the stellar surface gravity and temperature - also derived from the 3D simulations. Furthermore, to make our models consistent, we have calculated new opacity tables to match the atmospheric simulations. Here, we present the modified code and initial results on stellar evolution using it.
\end{abstract}

\section{Introduction}

Understanding stellar structure and evolution is one of the key ingredients in astrophysics. Perhaps the primary tool for doing so is evolutionary calculations of stellar structure, i.e. one-dimensional numerical models. These have been developed and tested through decades; as a result they are highly optimised and very efficient. However, in several aspects they are also highly simplified and can be improved. This work focuses on two of these: the use of a simple temperature stratification as part of an idealised atmosphere for the model and the treatment of stellar convection.

The artificial atmosphere is needed to supply the outer boundary conditions for stellar evolution codes in order to solve the differential equations of stellar structure (see e.g. [1]). The boundary conditions are obtained by integrating an analytical expression for the temperature as a function of optical depth - a so-called $T(\tau)$ relation. A general issue is that these relations are much too simple to capture the true stratification of stellar atmospheres.

In $1 \mathrm{D}$ models, convection is typically treated using a parametric description. The most common is the mixinglength theory (MLT) as introduced for stellar models by $[2,3]$ or some variant thereof. These parametric theories rely on rather crude assumptions, which are certainly not fulfilled in stars (see e.g. [4]). Furthermore, a free parameter (the mixing-length parameter, $\alpha_{\mathrm{MLT}}$ ) is "calibrated" against the Sun by some observational constraint, usually the solar radius. The solar value is then assumed to be valid for all stars across the HR diagram.

\footnotetext{
^e-mail: jakob@phys.au.dk
}

To improve the models, highly sophisticated threedimensional simulations of stellar atmospheres (see the next section) can be used in different ways. One approach is to patch the averaged 3D structure on top of a 1D model, as described by [5]. This of course requires the 1D model to match the 3D simulation perfectly.

In this work, we employ a different approach, where condensed information - specifically targeted at the two issues mentioned above - is extracted from the simulations and interpolated to any point between them. The advantage of this approach is that it can be used in stellar structure models for full evolutionary calculations; the patched models are static by nature. An implementation similar to this work, however not fully consistent, was attempted by [6].

In section 2 we review the 3D simulations used in this work and how useful information is extracted from them. Our implementation is described in detail in section 3, and finally we present a few initial results in section 4 .

\section{Simulations of Stellar Atmospheres}

Stellar atmospheres are very complex regions, where hydrodynamics and radiative transfer interact. For a better understanding of this important part of stars several authors have employed advanced 3D simulations, which account for both hydrodynamics and radiative transfer. These simulations have been used extensively to study the convection and granulation in the outer parts of our Sun (e.g. [7, 8]).

More recently, several groups have produced grids of 3D simulations to investigate the behaviour of stars different from our Sun. In this work, we will use the grid of simulations from Trampedach et al. [9], which consists of 
37 simulations at solar metallicity - the physical parameters (effective temperature, $T_{\text {eff }}$, and surface gravity, $\log g$ ) of the different simulations are shown in Figure 1, which is described in further details below.

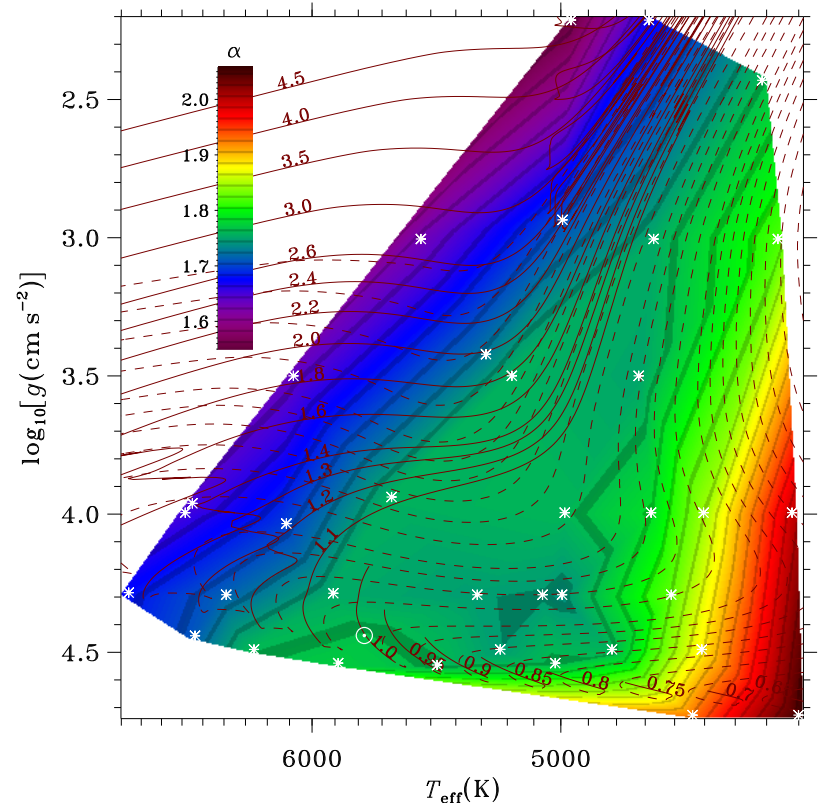

Figure 1: The grid of 3D simulations from [9] at solar metallicity. The simulation parameters are marked by white asterisks and the Sun by $\odot$. The coloured background shows the behaviour of $\alpha_{\mathrm{MLT}}$ as a function of $T_{\text {eff }}$ and $\log g$. The red lines represents evolutionary tracks, but they are unimportant for the present work.

[Reproduced with permission from [10], published by Oxford University Press on behalf of The Royal Astronomical Society. All rights reserved. Available online at http://dx.doi.org/10.1093/mnras/stu2084. This figure is not covered by the Open-Access licence of this publication. For permissions contact Journals.permissions@ouP.com]

\subsection{Condensed 3D Simulations}

For the purpose of stellar evolutionary calculations, the full (averaged) 3D structures cannot be used directly for several reasons. First of all, they only sample discrete points in the HR diagram, and it is not feasible to make the grids much denser since the simulations are extremely computationally expensive. ${ }^{1}$ Secondly, due to differences in physics (e.g. treatment of convection and turbulent pressure) they are not directly compatible with the current 1D models.

For these reasons, Trampedach et al. [11] have devised a way of distilling the 3D simulations to be easily usable in stellar structure models. In short, the temperature stratification is extracted from the simulations in the form of the (generalised) Hopf function, $q$, as a function of Rosseland mean optical depth, $\tau$. This is then used for reconstructing the photospheric transition, from optically thick to optically thin, of the 3D simulation in the 1D model.

Furthermore, it is possible to calibrate the mixinglength parameter, $\alpha_{\mathrm{MLT}}$, against the 3D simulations. This

\footnotetext{
${ }^{1}$ One could in principle interpolate between the full simulations, but such a scheme has yet to be developed.
}

correctly connects the entropy of the adiabatic layers deep in the star with the surface layers in the 3D atmosphere. Besides, the need to assume a solar-calibrated value for all stars is eliminated. This is done by [10] for all simulations in the grid - the actual procedure is very similar to the patching of 3D simulations to 1D models mentioned earlier. The corresponding $\alpha_{\mathrm{MLT}}$ 's are stored in the table with $q(\tau)$, and the interpolated behaviour as a function of $T_{\text {eff }}$ and $\log g$ is shown in Figure 1. It should be noted that the calibrated values are of course only strictly correct, if the stellar evolution code uses the same MLT-formulation as the 1D (envelope) code used for the calibration.

\section{Implementation}

We have implemented the results from $[10,11]$ (in the form of a table containing $q(\tau)$ and $\alpha_{\mathrm{MLT}}$ ) into the Garching Stellar Evolution Code (GARSTEC, see [12]). A simple sketch of the implementation is shown in Figure 2.

The general principle is that the $q(\tau)$ and $\alpha_{\mathrm{MLT}}$ corresponding to $T_{\text {eff }}$ and $\log g$ of the star are found by interpolation $^{2}$ in the grid and stored in the program. In each iteration of the code, these values are updated to always match the current stellar parameters.

The quantities are then supplied to the different parts of the code; the atmospheric integration (providing the outer boundary conditions) receives the $q(\tau)$ and the routine handling MLT-convection uses the new $\alpha_{\text {MLT }}$. Moreover, for reasons explained below, the convection module also needs access to $q(\tau)$.

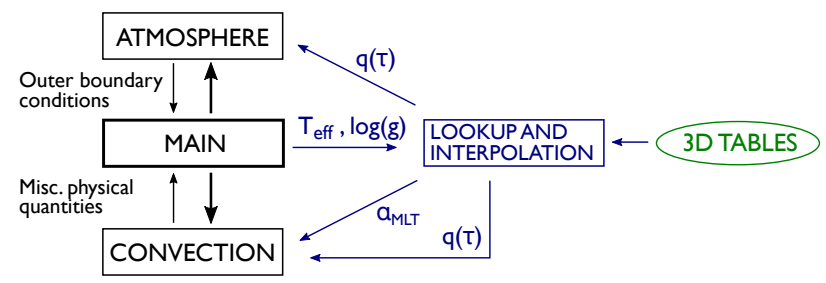

Figure 2: Schematic overview of our implementation marked in blue. The black represents modules in GARSTEC, while the green circle marks the tables from [10,11]. Details are given in the text.

\subsection{Changes to a Stellar Model}

How a stellar structure model is modified by our implementation is outlined in Figure 3.

In the atmosphere, the generalised Hopf function from the simulations, $q(\tau)$, is used to provide the stratification instead of the standard analytical Eddington atmosphere, $T_{\text {edd }}(\tau)$. Assuming radiative equilibrium, the new temperature structure is found from $q(\tau)$ using

$$
\frac{4}{3}\left(\frac{T}{T_{\text {eff }}}\right)^{4}=q(\tau)+\tau,
$$

\footnotetext{
${ }^{2}$ We use the interpolation scheme supplied by [10], which is a linear interpolation over a Thiessen triangulation of the irregular grid of simulations.
} 


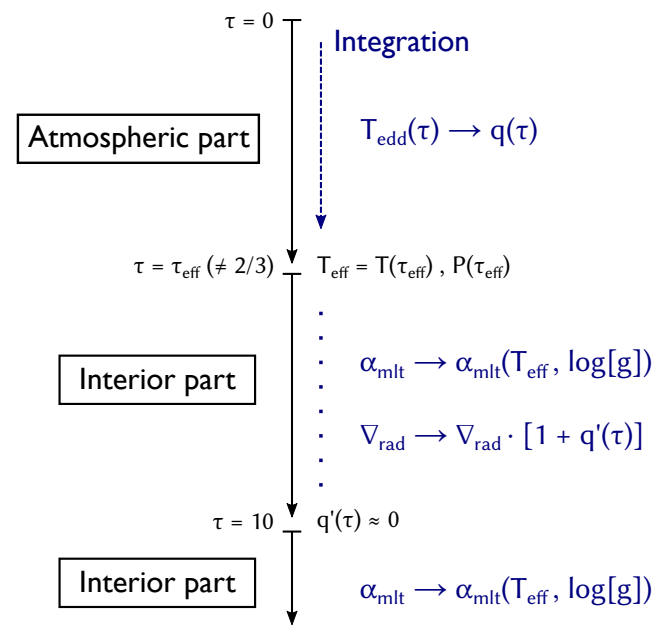

Figure 3: The changes to a stellar structure model as a result of our implementation. In the atmosphere, a different temperature stratification is used and the integration point is altered. In the outer, convective parts of the interior model, the radiative gradient is modified according to eq. (3). Everywhere in the interior of the star, the calibrated $\alpha_{\mathrm{MLT}}\left(T_{\text {eff }}, \log g\right)$ is used.

as explained by [11, eq. 9].

Another alteration is the bottom point of the atmosphere, i.e. where the integration is stopped - note that in stellar structure models this point is usually defined to have $T=T_{\text {eff }}$. The Eddington atmosphere is anchored at $\tau=2 / 3$, but that is not the case for the relations extracted from 3D simulations. Thus before the actual integration, we find the point $\tau_{\text {eff }}$ in the interpolated temperature structure where $T=T_{\text {eff. }}{ }^{3}$

Moving on to the interior part of the model, the first change is the use of the $3 \mathrm{D}$-calibrated variable mixinglength parameter, $\alpha_{\mathrm{MLT}}\left(T_{\text {eff }}, \log g\right)$, throughout the model. We are not using the $\alpha_{\mathrm{MLT}}$, grid directly as provided from the tables, but rather using it differentially as recommended by $[11,13]$. In other words, we introduce a scaling factor

$$
\alpha_{\mathrm{MLT}}\left(T_{\mathrm{eff}}, \log g\right)=\frac{\alpha_{\odot}}{\alpha_{\odot, \text { grid }}} \cdot \alpha_{\mathrm{MLT}, \text { grid }}\left(T_{\mathrm{eff}}, \log g\right),
$$

where $\alpha_{\odot}$ is what we obtain from a solar calibration in GARSTEC and $\alpha_{\odot \text {, grid }}$ is the value given for the solar model in the grid. This ensures that the solar model is calibrated to the correct radius with this variable $\alpha_{\mathrm{MLT}}$.

When using Eq. (1) for the temperature structure, the temperature gradient for a stratification in radiative equilibrium (i.e. no convective flux), $\nabla_{\mathrm{rad}} \equiv(\partial \ln T / \partial \ln p)_{\mathrm{rad}}$, needs to be corrected from its optically deep value of $\tilde{\nabla}_{\text {rad }}$, as

$$
\nabla_{\mathrm{rad}}=\tilde{\nabla}_{\mathrm{rad}} \cdot\left[1+q^{\prime}(\tau)\right],
$$

according to [11, eq. 35]. Here $q^{\prime}(\tau)$ is the derivative of the Hopf function with respect to $\tau$ and $\tilde{\nabla}_{\text {rad }}$ is the usual expression for the radiative gradient, based on the diffusion approximation. This modification is applied before entering the MLT routine of GARSTEC; thus the resulting

\footnotetext{
${ }^{3}$ The value is typically $\tau_{\text {eff }} \simeq 0.5$, and $T_{\text {eff }}$ is here the parameter of the (interpolated) $3 \mathrm{D}$ simulation.
}

temperature gradient $\nabla$ is properly corrected. ${ }^{4}$ We apply this correction factor until $\tau=10$, since $q^{\prime}(\tau)$ at larger depth is always below $10^{-4}$ (and typically below $10^{-5}$ ), where the adiabatic layers are reached.

\subsection{Important Remarks}

At this point it important to stress that it is crucial for the stellar evolution code to use the same microphysics as the 3D simulations for consistency.

To match the simulations, we use the MHD equation of state (EOS) [14-16]. To cover the necessary range of temperatures, we complement it with OPAL EOS [17] at higher temperatures.

For the 3D simulations, [11] calculated their own atmospheric (i.e. low-temperature) opacities, which in the envelope model used for the matching was merged with interior opacities from the Opacity Project (OP, see [18]). We have calculated similar opacity tables for the specific solar mixture used in the simulations (see [9]) using the atmospheric opacities provided by the author and the available OP data.

\section{Initial Results}

The natural test of our implementation is to calculate evolutionary tracks and compare to a standard evolution. In this context standard means: using Eddington atmosphere and a constant solar-calibrated $\alpha_{\text {MLT }}$.

We did a solar calibration (using the opacities explained above) and obtained $\alpha_{\odot}=1.742$ for the traditional Eddington atmosphere and $\alpha_{\odot}=1.870$ for our new atmosphere. In the latter case, eq. (2) is utilised to obtain the scaled $\alpha_{\mathrm{MLT}}$ used in the calculation. ${ }^{5}$

The results for a $1.0 M_{\odot}$ and a $1.4 M_{\odot}$ star are shown in Figures 4 and 5, respectively. It can be seen that for the first track the temperature differences are minuscule except at the RGB. For the heavier star, the differences are larger, but the tracks are actually closer to each other higher up the RGB.

Regarding the evolutionary pace of the models, it is also quite similar. At the end of the main sequence (i.e. where central hydrogen is exhausted) the age difference is less than 0.01 Gyr between the tracks - similar for the end of the calculation at $\log g=2.4$. However, using a more observational "definition" of the turn-off - the point of the track where $T_{\text {eff }}$ reaches maximum - there is a difference in age of $\sim 0.5 \mathrm{Gyr}$ for the $1.0 M_{\odot}$ model. This is an interesting aspect of the new atmosphere, which we will investigate further.

We plan to next look for differences in interior structure (if any) between models with our implementation and the standard calculation. It will be very interesting to use asteroseismology (see e.g. [19]) and compare the oscillation frequencies between modified and unmodified models. We will follow this line of investigation in a coming paper.

\footnotetext{
${ }^{4}$ The modified $\nabla_{\text {rad }}$ is of course also used when checking for convection with the Schwarzschild criterion.

${ }^{5}$ The solar value of the grid from [11] is $\alpha_{\odot \text {, grid }}=1.767$, which gives a scaling factor of 1.058 to be applied to the interpolated $\alpha_{\mathrm{MLT}}\left(T_{\mathrm{eff}}, \log g\right)$.
} 


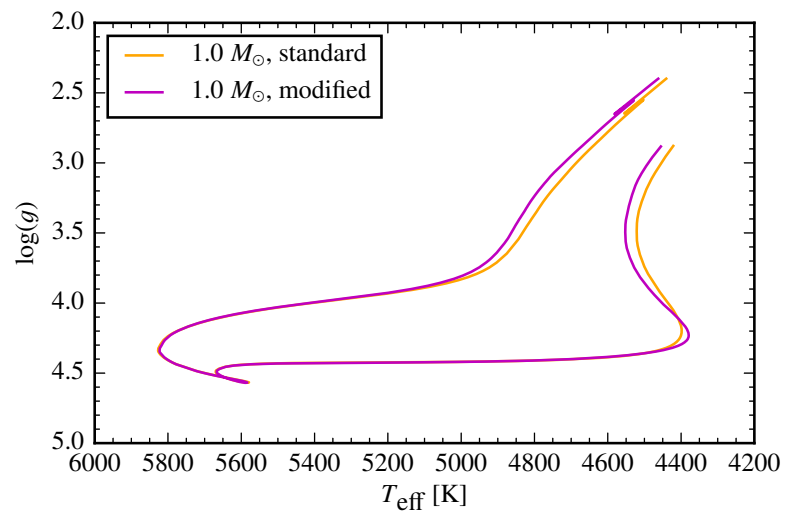

Figure 4: Evolutionary track of a $1.0 M_{\odot}$ star from the pre main sequence to $\log g=2.4$. The standard track uses a Eddington atmosphere and solar calibrated $\alpha_{\text {MLT }}$. The modified track uses our implementation of $q(\tau)$ and $\alpha_{\mathrm{MLT}}\left(T_{\mathrm{eff}}, \log g\right)$.

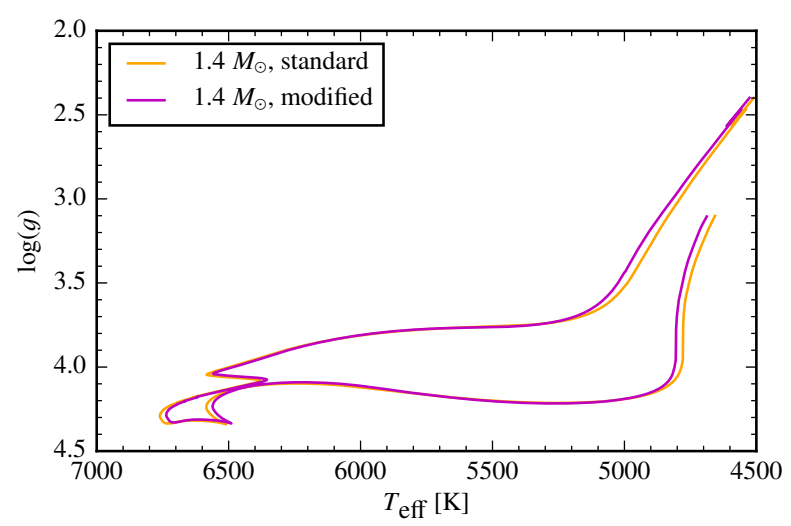

Figure 5: As Figure 4, but for a $1.4 M_{\odot}$ star.

\section{Conclusion}

We have successfully implemented $T(\tau)$ relations extracted from $3 \mathrm{D}$ simulations and the associated modifications to the thermal gradients in the Garching Stellar Evolution Code. Furthermore, we have also modified the code to use a variable mixing-length parameter, $\alpha_{\mathrm{MLT}}\left(T_{\mathrm{eff}}, \log g\right)$, obtained from calibration against the $3 \mathrm{D}$ simulations. For consistency we have calculated new opacity tables to match those used in the simulations, and we use the same equation of state.

In a future work we will investigate the effect of our modifications on the interior structure of the models and more specifically the oscillation frequencies. We will also investigate the impact on the derived age when comparing models to observations.

\section{Acknowledgements}

Funding for the Stellar Astrophysics Centre (SAC) is provided by The Danish National Research Foundation (Grant agreement no.: DNRF106).

\section{References}

[1] R. Kippenhahn, A. Weigert, A. Weiss, Stellar Structure and Evolution, second edition edn. (Springer, 2012)

[2] E. Vitense, Zeitschrift für Astrophysik 32, 135 (1953)

[3] E. Böhm-Vitense, Zeitschrift für Astrophysik 46 (1958)

[4] R. Trampedach, Astrophysics and Space Science 328, 213 (2010)

[5] C.S. Rosenthal, J. Christensen-Dalsgaard, Å. Nordlund, R.F. Stein, R. Trampedach, Astronomy and Astrophysics 351, 689 (1999)

[6] M. Salaris, S. Cassisi, Astronomy \& Astrophysics 577, A60 (2015)

[7] R.F. Stein, ̊. Nordlund, The Astrophysical Journal 342, L95 (1989)

[8] R.F. Stein, Å. Nordlund, The Astrophysical Journal 499, 914 (1998)

[9] R. Trampedach, M. Asplund, R. Collet, Å. Nordlund, R.F. Stein, The Astrophysical Journal 769, 18 (2013)

[10] R. Trampedach, R.F. Stein, J. Christensen-Dalsgaard, $\AA$. Nordlund, M. Asplund, Monthly Notices of the Royal Astronomical Society 445, 4366 (2014)

[11] R. Trampedach, R.F. Stein, J. Christensen-Dalsgaard, A. Nordlund, M. Asplund, Monthly Notices of the Royal Astronomical Society 442, 805 (2014)

[12] A. Weiss, H. Schlattl, Astrophysics and Space Science 316, 99 (2008)

[13] H.G. Ludwig, B. Freytag, M. Steffen, Astronomy \& Astrophysics 346, 111 (1999)

[14] D.G. Hummer, D. Mihalas, The Astrophysical Journal 331, 794 (1988)

[15] D. Mihalas, W. Dappen, D.G. Hummer, The Astrophysical Journal 331, 815 (1988)

[16] W. Däppen, D. Mihalas, D.G. Hummer, B.W. Mihalas, The Astrophysical Journal 332, 261 (1988)

[17] F.J. Rogers, F.J. Swenson, C.A. Iglesias, The Astrophysical Journal 456, 902 (1996)

[18] N.R. Badnell, M.A. Bautista, K. Butler, F. Delahaye, C. Mendoza, P. Palmeri, C.J. Zeippen, M.J. Seaton, Monthly Notices of the Royal Astronomical Society 360, 458 (2005)

[19] C. Aerts, J. Christensen-Dalsgaard, D.W. Kurtz, Asteroseismology, Astronomy and Astrophysics Library (Springer Science+Business Media, 2010) 